\title{
Constraints on the $S=-1$ meson-baryon interaction at NLO
}

\author{
A. Feijoo ${ }^{1, a}$, V.K. Magas ${ }^{1}$, and A. Ramos ${ }^{1}$ \\ ${ }^{1}$ Departament de Física Quàntica i Astrofísica and Institut de Ciències del Cosmos, Universitat de \\ Barcelona, Martí Franquès 1, E08028 Barcelona, Spain
}

\begin{abstract}
This work contains a study of the meson-baryon interaction in the $S=-1$ sector by means of a chiral $S U(3)$ Lagrangian up to next-to-leading order (NLO) and implementing unitarization in coupled channels. In order to get more reliable values of the parameters which are present in the model, we performed several fits which take a large set of experimental scattering data in different two-body channels, threshold branching ratios, and the precise SIDDHARTA values of the energy shift and width of kaonic hidrogen into consideration. In previous studies, we had shown that the $K^{-} p \rightarrow K \Xi$ reactions are especially sensitive to the next to Weinberg-Tomozawa (WT) corrections in the hierarchy. In addition, we pointed out the need to employ processes which are described by pure isospin amplitudes as a tool to discern which models are more realistic among those which give small values for the $\chi^{2}$ in the fits. Following the former suggestion, we present results which include data from $K^{-} p \rightarrow \eta \Lambda, \eta \Sigma$ reactions which have pure isospin $I=0$ and $I=1$ component respectively. Finally, to check the goodness of the new obtained parametrization of the model, we present a prediction for another process that filters the $I=1$ isospin component: the pure $I=1 K_{L}^{-} p \rightarrow K^{+} \Xi^{0}$ reaction which could be measured at the proposed secondary $K_{L}^{0}$ beam at Jlab.
\end{abstract}

\section{Introduction}

Chiral Perturbation Theory (ChPT) is an effective theory which has proved to be appropriate to describe the interaction of hadrons at low energies where Quantum Chromodynamics (QCD) fails in describing the strong interaction by means of a perturbative treatment. As it is well known, in ChPT, the dynamics is described by arranging an $S U(3)$ Effective Lagrangian as an expansion on the powers of the external momenta of the Nambu-Goldstone bosons over a typical scale $\left(\Lambda_{C h P T} \approx 1 \mathrm{GeV}\right)$. This Effective Lagrangian has to be invariant under the same symmetries as QCD, among them, the invariance under chiral symmetry plays key role [1-3]. Being more specific, the spontaneous breaking of the chiral symmetry gives rise to the corresponding Goldstone bosons which are identified with the pseudoscalar mesons in this theoretical framework.

Although the strong constraints imposed by chiral-symmetry-breaking allow one to take into consideration processes which are restricted to the energy limit given by $\Lambda_{C h P T}$, Effective Chiral theories have drawbacks when a resonance appears in the region of interest because it is associated to a pole in the scattering amplitude which cannot be reproduced by means of a perturbative expansion. One can

ae-mail: feijoo@fqa.ub.edu 
then resort to nonperturbative schemes, which allow one to get around this difficulty while maintaining the predictive power of the theory.

Unitarized Chiral Perturbation Theory (UChPT), which combines chiral dynamics with unitarization techniques in coupled channels, has been shown to be a very powerful tool to describe the hadron interaction including the dynamical generation of bound-states and resonances (see [4] and references therein). This scheme is based on two guiding principles: the inherited symmetries from QCD, particularly (spontaneously broken) chiral symmetry, and general properties of the scattering amplitude such as unitarity and analiticity. Besides the mentioned advantages, UChPT permits an extension of the validity of ChPT to higher energies.

The effectiveness of UChPT can be ilustrated by the description of the $\Lambda(1405)$ resonance which is located $27 \mathrm{MeV}$ below the $\bar{K} N$ threshold. In [5], the dynamical generation of $\Lambda(1405)$ was formulated, for the first time, in terms of the unitarized chiral meson-baryon interaction in coupled channels almost 40 years after its dynamical origin was pointed out [6]. A lot of activity was awaken by this success in the comunity, which analyzed the effects of including a complete basis of meson-baryon channels, differences in the regularization of the equations, s- and u-channel Born terms in the Lagrangian, next-to-leading (NLO) contributions, etc ... [7-16].

The rising number of more precise measurements such as the energy shift and width of the 1s state in kaonic hydrogen by the SIDDHARTA collaboration [17] at DAФNE have renewed the interest in this topic in these last few years. As a response to this emerging experimental activity, the theoretical models have been revisited [18-21].

In this context, and taking into account the descriptive power of the UChPT approach, the $S=-1$ sector offers us a good chance to extract information about those parameters of the model which are not well constrained. One should bear in mind that these parameters are not fixed by the symmetries on which the theory is based, but the values come from fitting procedures to the experimental data.

From the studies of the meson-baryon interaction in s-wave approximation [8-16, 18-21], it can be concluded that the significant term that allows one to get a good agreement with the experimental data is the Weinberg-Tomozawa (WT) one, which is of order $O\left(p^{2}\right)$, and the addition of other terms such as the direct and crossed Born terms $\left(O\left(p^{2}\right)\right)$ as well as the NLO terms $\left(O\left(p^{4}\right)\right)$ only play a finetuning role. The corresponding diagrams are represented in figure 1. At this point, it is important to highlight that the fits carried out to develop these models were accommodated to the two-body cross sections of $\bar{K} N$ scattering into $\pi \Sigma, \bar{K} N, \pi \Lambda$ states, and also $\eta \Lambda$ in the particular case of [19].

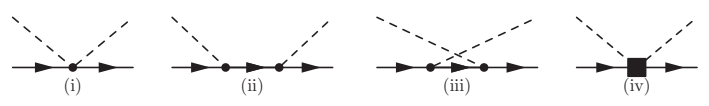

Figure 1. Feynman diagrams for the meson-baryon interaction: Weinberg-Tomozawa term (i), direct and crossed Born terms (ii) and (iii), and NLO terms (iv). Dashed (solid) lines represent the pseudoscalar octet mesons (octet baryons).

Until [22], the reproduction of $K \Xi$ production cross section had not been studied employing chiral models. The work of [22] focused on the $K^{-} p \rightarrow K \Xi$ reactions because the WT term has a zero direct contribution to the scattering amplitude for these reactions and the strength coming from the rescattering terms due to the coupled channels is not sufficient to reproduce the experimental scattering data as it is shown therein. This fact confers an interesting role to the $K^{-} p \rightarrow K \Xi$ reaction as a privileged framework in the $S=-1$ sector to get information about the next terms in the hierachy beyond the WT one, namely the direct and cross Born terms and the NLO term. From a leading order calculation, the authors of [9] pointed out that the contribution of the $s$ and $u$ Born diagrams are almost 
negligible at energies around $1.3 \mathrm{GeV}$ while their magnitude increases with energy reaching $\approx 20 \%$ of the dominant WT contribution. Despite the previous analysis, one finds that the contribution of the cross and direct Born terms is very moderate for studies which were based on lagrangians extended to NLO $[15,18,21]$. Assuming this and for simplicity, the Born contributions were disregarded in our study of [22] leaving the NLO term as the significant one in order to reach good agreement between the theoretical predictions and the experimental data in the $K \Xi$ channels. We fitted our model to the branching ratios at $K^{-} p$ threshold [24, 25], the precise SIDDHARTA values of the shift and width of the 1s state of the kaonic hydrogen [17] and the cross-sections for the different two body channels $(\pi \Sigma, \pi \Lambda, \bar{K} N, K \Xi)[26,27]$. After comparing different fitting procedures, the sensitivity of the $K^{-} p \rightarrow K \Xi$ reactions to the NLO term of the chiral Lagrangian was demonstrated and, therefore, more reliable values of the low energy constants were obtained. Moreover, in order to study the accuracy and stability of our parameters, we included high spin and high mass resonances applying the RaritaSchwinger method [28-30]. Based on the phenomenological study of [30], we chose as candidates the $\Sigma(2030)$ and $\Sigma(2250)$ resonances and we achieved very good agreement with the experimental data. Finally, as we had expected, we got a more precise and trustable determination of the corresponding NLO parameters. This last result could be explained because the inclusion of these resonant terms simulate the contributions of higher angular momenta of the other channels, via rescattering, in the energy regim above the $K \Xi$ threshold and, consequently, the parameters of the model get relaxed avoiding a possible overestimation of their values.

The natural next stage of our work was to determine whether the assumption of non significant contribution of the Born diagrams in the interaction kernel is still realistic once the $K \Xi$ channels are included in the fits. Our study of [31], merely employing a chiral model which takes into account the Born terms, revealed the particular importance of these diagrams in the $K \Xi$ channels which translated into significant modifications of the NLO parameters. Contrary to expectations, the resulting effects of the inclusion of new relevant terms did not improve the reproduction of the experimental data, since we got very similar values of the $\chi^{2}$ in comparison with the ones coming from the models in [22]. The relevance of the Born terms becomes more visible in the isospin projected amplitudes of the $K^{-} p \rightarrow K \Xi$ reaction which differ substantially from what was found for the models that ignored the Born terms, see figure 5 in [31]. This fact points towards the idea that processes that filter a single isospin component are essential requirement to get much more reliable values for the NLO coefficients.

For instance, the weak decay of the $\Lambda_{b}$ into $J / \psi K \Xi$ and $J / \psi \eta \Lambda$ states was studied in [32]. These processes involve an elementary weak transition at the quark level which proceeds via the creation of a $J / \psi$ meson and excited sud system with $I=0$ that hadronizes into a final meson-baryon pair in $I=0$ state. Thus, an experimental determination of these decays would contribute to a better understanding of the chiral dynamics at higher energies. The insights of this study are summarized in the contribution to the same proceedings by V.K. Magas [33]. In a complementary manner to the $\Lambda_{b}$ decay, the obtention of experimental data from a pure $I=1 \bar{K} N \rightarrow K \Xi$ reaction could supply significant information. The recent proposal [34] of creating a secondary $K_{L}^{0}$ beam at Jlab offers a great opportunity for measuring the $K_{L}^{0} p \rightarrow K^{+} \Xi^{0}$ reaction. We include a prediction for this reaction in the present work. Nevertheless the inclusion of the experimental data from $K^{-} p \rightarrow \eta \Lambda, \eta \Sigma^{0}$ reactions in the fitting procedure is the most natural filtering process that might be provided. These two reactions are very useful to discriminate possible ambiguities in the isospin distributions since they are pure $I=0$ and $I=1$ processes, respectively. The motivation of the present study is to implement these isospin filtering scattering processes into our set of experimental data in order to obtain more trustable and reliable values of the NLO coefficients, hence improving over our previous models of the $S=-1$ interaction. 


\section{Chiral unitary approach}

The $S U(3)$ chiral effective Lagrangian at NLO for describing the meson-baryon interaction is,

$$
\mathcal{L}_{\phi B}^{e f f}=\mathcal{L}_{\phi B}^{(1)}+\mathcal{L}_{\phi B}^{(2)}
$$

being $\mathcal{L}_{\phi B}^{(1)}$ and $\mathcal{L}_{\phi B}^{(2)}$ the most general forms of the contribution at leading order and the contribution at next-to-leading order of the meson-baryon interaction Lagrangian, respectively, and which are expressed as follows

$$
\begin{aligned}
\mathcal{L}_{\phi B}^{(1)}= & i\left\langle\bar{B} \gamma_{\mu}\left[D^{\mu}, B\right]\right\rangle-M_{0}\langle\bar{B} B\rangle-\frac{1}{2} D\left\langle\bar{B} \gamma_{\mu} \gamma_{5}\left\{u^{\mu}, B\right\}\right\rangle-\frac{1}{2} F\left\langle\bar{B} \gamma_{\mu} \gamma_{5}\left[u^{\mu}, B\right]\right\rangle \\
\mathcal{L}_{\phi B}^{(2)}= & b_{D}\left\langle\bar{B}\left\{\chi_{+}, B\right\}\right\rangle+b_{F}\left\langle\bar{B}\left[\chi_{+}, B\right]\right\rangle+b_{0}\langle\bar{B} B\rangle\left\langle\chi_{+}\right\rangle+d_{1}\left\langle\bar{B}\left\{u_{\mu},\left[u^{\mu}, B\right]\right\}\right\rangle+d_{2}\left\langle\bar{B}\left[u_{\mu},\left[u^{\mu}, B\right]\right]\right\rangle \\
& +d_{3}\left\langle\bar{B} u_{\mu}\right\rangle\left\langle u^{\mu} B\right\rangle+d_{4}\langle\bar{B} B\rangle\left\langle u^{\mu} u_{\mu}\right\rangle .
\end{aligned}
$$

In both equations, the symbol $\langle\ldots\rangle$ stands for the trace in flavor space and $U(\phi)=u^{2}(\phi)=$ $\exp \left(\sqrt{2} i \frac{\phi}{f}\right)$, with $\phi$ the unitary $3 \times 3$ matrix for the pseudoscalar meson octet $(\pi, K, \eta)$, which enters the Lagrangian in the combination $u_{\mu}=i u^{\dagger} \partial_{\mu} U u^{\dagger}$. Thus, the parameter $f$ corresponds to the pseudoscalar decay constant in the chiral limit. The unitary $3 \times 3 \mathrm{~B}$ matrix collects the octet baryon fields $(N, \Lambda, \Sigma, \Xi)$. In eq. (2), $M_{0}$ is the common baryon octet mass in the chiral limit, and the constants $D, F$ denote the axial vector couplings of the baryons to the mesons. Furthermore, $\left[D_{\mu}, B\right]=\partial_{\mu} B+\left[\Gamma_{\mu}, B\right]$ stands for the covariant derivative with $\Gamma_{\mu}=\left[u^{\dagger}, \partial_{\mu} u\right] / 2$ being the chiral connection. In addition, in eq. (3), we find $\chi_{+}=2 B_{0}\left(u^{\dagger} \mathcal{M} u^{\dagger}+u \mathcal{M} u\right)$ which breaks chiral symmetry explicitly via the quark mass matrix $\mathcal{M}=\operatorname{diag}\left(m_{u}, m_{d}, m_{s}\right)$ and $B_{0}=-\langle 0|\bar{q} q| 0\rangle / f^{2}$ which relates to the order parameter of spontaneously broken chiral symmetry. As we see, there are 7 parameters at NLO, namely the low energy constants $b_{D}, b_{F}, b_{0}$ and $d_{i}(i=1, \ldots, 4)$, which will be fitted to experimental data.

The Weinberg-Tomozawa (WT) term corresponds to the diagram (i) in figure 1 and, in a nonrelativistic limit, it reads:

$$
V_{i j}^{W T}=-C_{i j} \frac{1}{4 f^{2}} \mathcal{N}_{i} \mathcal{N}_{j}\left(\sqrt{s}-M_{i}-M_{j}\right),
$$

where $\sqrt{s}$ is the total energy of the meson-baryon system in the center-of-mass frame. As we see, the WT term depends only on one parameter - the pion decay constant $f$, which is well known experimentally, $f_{\pi}=92.4 \mathrm{MeV}$. However in $\mathrm{U} \chi \mathrm{PT}$ calculations this parameter is usually taken to be larger than the experimental value, ranging from $f=1.15 f_{\pi}$ to $f=1.36 f_{\pi}[7-16,18-21]$, meaning to be a sort of average over the decay constants of the mesons involved in the various coupled channels. We will leave it as a free parameter of our fits.

The indices $(i, j)$ cover all the initial and final channels, which, in the case of strangeness $S=-1$ and charge $Q=0$ explored here, amount to ten: $K^{-} p, \bar{K}^{0} n, \pi^{0} \Lambda, \pi^{0} \Sigma^{0}, \pi^{-} \Sigma^{+}, \pi^{+} \Sigma^{-}, \eta \Lambda, \eta \Sigma^{0}, K^{+} \Xi^{-}$ and $K^{0} \Xi^{0}$. The matrix of coefficients $C_{i j}$ can be found in table VII of [22]. The normalization factor $\mathcal{N}_{i}$ is defined as $\mathcal{N}_{i}=\sqrt{\left(M_{i}+E_{i}\right) /\left(2 M_{i}\right)}$, with $M_{i}$ and $E_{i}$ being, respectively, the mass and energy of the baryon of the $i-t h$ channel.

We next consider the s-wave projection and the non relativistic assumption for the the so called Born diagrams. The direct Born term, shown in figure 1 (ii), is given by

$$
V_{i j}^{D}=-\sum_{k=1}^{8} \frac{C_{\bar{i} i, k}^{(\text {Born })} C_{\bar{j} j, k}^{(\text {Born })}}{12 f^{2}} \mathcal{N}_{i} \mathcal{N}_{j} \frac{\left(\sqrt{s}-M_{i}\right)\left(\sqrt{s}-M_{k}\right)\left(\sqrt{s}-M_{j}\right)}{s-M_{k}^{2}},
$$


where the $k$ label refers to the intermediate baryon involved in the process. The coefficients $C_{\bar{i} i, k}^{\text {(Born) }}$ can be found in [15] and they depend on the axial vector constants $D$ and $F$. In the same way, the crossed Born term, shown in figure 1 (iii), reads

$$
\begin{aligned}
V_{i j}^{C}= & \sum_{k=1}^{8} \frac{C_{\bar{j} k, i}^{\text {(Born) }} C_{\bar{i} k, j}^{\text {(Born) }}}{12 f^{2}} \mathcal{N}_{i} \mathcal{N}_{j} \times\left[\sqrt{s}+M_{k}-\frac{\left(M_{i}+M_{k}\right)\left(M_{j}+M_{k}\right)}{2\left(M_{i}+E_{i}\right)\left(M_{j}+E_{j}\right)}\left(\sqrt{s}-M_{k}+M_{i}+M_{j}\right)\right. \\
& +\frac{\left(M_{i}+M_{k}\right)\left(M_{j}+M_{k}\right)}{4 q_{i} q_{j}}\left\{\sqrt{s}+M_{k}-M_{i}-M_{j}-\frac{s+M_{k}^{2}-m_{i}^{2}-m_{j}^{2}-2 E_{i} E_{j}}{2\left(M_{i}+E_{i}\right)\left(M_{j}+E_{j}\right)}\left(\sqrt{s}-M_{k}+M_{i}+M_{j}\right)\right\} \\
& \left.\times \ln \frac{s+M_{k}^{2}-m_{i}^{2}-m_{j}^{2}-2 E_{i} E_{j}-2 q_{i} q_{j}}{s+M_{k}^{2}-m_{i}^{2}-m_{j}^{2}-2 E_{i} E_{j}+2 q_{i} q_{j}}\right]
\end{aligned}
$$

where $q_{i}, q_{j}$ are the center-of-mass (CM) three-momenta in the corresponding $i, j$ channels, and $m_{i}, m_{j}$ denote the corresponding meson masses.

From the Lagrangian $\mathcal{L}_{\phi B}^{(2)}$ one can derive the meson-baryon interaction kernel at NLO:

$$
V_{i j}^{N L O}=\frac{1}{f^{2}} \mathcal{N}_{i} \mathcal{N}_{j}\left[D_{i j}-2\left(\omega_{i} \omega_{j}+\frac{q_{i}^{2} q_{j}^{2}}{3\left(M_{i}+E_{i}\right)\left(M_{j}+E_{j}\right)}\right) L_{i j}\right],
$$

where $\omega_{i}, \omega_{j}$ are the meson energies involved in the transition amplitude. The $D_{i j}$ and $L_{i j}$ coefficients depend on the NLO parameters $b_{0}, b_{D}, b_{F}, d_{1}, d_{2}, d_{3}$ and $d_{4}$ and are given in table VIII of [22].

The final interaction kernel employed in this work is expressed as the sum:

$$
V_{i j}=V_{i j}^{W T}+V_{i j}^{D}+V_{i j}^{C}+V_{i j}^{N L O}
$$

In the introduction, it has been mentioned that the presence of resonances makes ChPT inapplicable because we can not reproduce the proper pole structure of the amplitude by means of a perturbative expansion. In particular, the $\bar{K} N$ interaction is strong enough to produce the $\Lambda(1405)$ as a quasi-bound. We can adress then a non-perturbative resummation by solving the Bethe-Salpeter equation in coupled channels using the interaction kernel derived from the chiral Lagrangian, i. e. eq. (8). This unitarization technique can be carried out by converting a complex system of integral equations into a coupled-channel algebraic equation system after using an on shell factorization $[8,35]$. Finally, the matrix form for the scattering amplitude reads:

$$
T_{i j}=\left(1-V_{i l} G_{l}\right)^{-1} V_{l j}
$$

where $V_{i j}$ is the interaction kernel (eq. (8)), for a given starting i-channel and an outgoing j-channel, and $G_{l}$ is the loop function, which needs to be regularized. We use dimensional regularization, which gives:

$$
G_{l}=\frac{2 M_{l}}{(4 \pi)^{2}}\left\{a_{l}+\ln \frac{M_{l}^{2}}{\mu^{2}}+\frac{m_{l}^{2}-M_{l}^{2}+s}{2 s} \ln \frac{m_{l}^{2}}{M_{l}^{2}}+\frac{q_{\mathrm{cm}}}{\sqrt{s}} \ln \left[\frac{\left(s+2 \sqrt{s} q_{\mathrm{cm}}\right)^{2}-\left(M_{l}^{2}-m_{l}^{2}\right)^{2}}{\left(s-2 \sqrt{s} q_{\mathrm{cm}}\right)^{2}-\left(M_{l}^{2}-m_{l}^{2}\right)^{2}}\right]\right\}
$$

where $M_{l}$ and $m_{l}$ are the baryon and meson masses of the " $l$ " channel, $\mu$ is the dimensional regularization scale, and $a_{l}$ are the so called subtraction constants, which are used as free parameters and fitted to the data. 
Table 1. Values of the parameters and the corresponding $\chi_{\text {d.o.f. }}^{2}$, defined in eq. (26) of [22], for the different fits described in the text. The subtraction constants are taken at a regularization scale $\mu=1 \mathrm{GeV}$. The error bars of the parameters are those given by the MINUIT minimization procedure.

* The higher value of the $\chi_{\text {d.o.f. }}^{2}$ could mislead the reader, but one should keep in mind that 58 additional experimental points from the $\eta$ channels had been included in the fitting procedure.

\begin{tabular}{lccc}
\hline & WT+NLO & WT+NLO+Born & $\begin{array}{c}\text { WT+NLO+Born } \\
\text { called NLO in [22] }\end{array}$ \\
\hline$a_{\bar{K} N}\left(10^{-3}\right)$ & $6.55 \pm 0.63$ & $1.77 \pm 2.38$ & $1.27 \pm 0.12$ \\
$a_{\pi \Lambda}\left(10^{-3}\right)$ & $54.8 \pm 7.5$ & $55.2 \pm 13.5$ & $-6.1 \pm 12.9$ \\
$a_{\pi \Sigma}\left(10^{-3}\right)$ & $-2.29 \pm 1.89$ & $2.33 \pm 3.17$ & $0.68 \pm 1.43$ \\
$a_{\eta \Lambda}\left(10^{-3}\right)$ & $-14.2 \pm 12.7$ & $8.00 \pm 5.04$ & $-0.67 \pm 1.06$ \\
$a_{\eta \Sigma}\left(10^{-3}\right)$ & $-5.17 \pm 0.07$ & $6.5 \pm 20.6$ & $8.00 \pm 3.26$ \\
$a_{K \Xi}\left(10^{-3}\right)$ & $27.0 \pm 7.8$ & $-9.04 \pm 3.63$ & $-2.51 \pm 0.99$ \\
$f / f_{\pi}$ & $1.20 \pm 0.01$ & $1.21 \pm 0.03$ & $1.20 \pm 0.03$ \\
$b_{0}\left(\mathrm{GeV}^{-1}\right)$ & $-1.21 \pm 0.01$ & $-0.70 \pm 0.23$ & $0.13 \pm 0.04$ \\
$b_{D}\left(\mathrm{GeV}^{-1}\right)$ & $0.05 \pm 0.04$ & $0.31 \pm 0.20$ & $0.12 \pm 0.01$ \\
$b_{F}\left(\mathrm{GeV}^{-1}\right)$ & $0.26 \pm 0.15$ & $0.65 \pm 0.41$ & $0.21 \pm 0.02$ \\
$d_{1}\left(\mathrm{GeV}^{-1}\right)$ & $-0.11 \pm 0.06$ & $0.17 \pm 0.26$ & $0.15 \pm 0.03$ \\
$d_{2}\left(\mathrm{GeV}^{-1}\right)$ & $0.65 \pm 0.02$ & $0.17 \pm 0.11$ & $0.13 \pm 0.03$ \\
$d_{3}\left(\mathrm{GeV}^{-1}\right)$ & $2.85 \pm 0.04$ & $0.37 \pm 0.16$ & $0.30 \pm 0.02$ \\
$d_{4}\left(\mathrm{GeV}^{-1}\right)$ & $-2.10 \pm 0.02$ & $0.01 \pm 0.09$ & $0.25 \pm 0.03$ \\
$D$ & - & $0.90 \pm 0.10$ & $0.70 \pm 0.16$ \\
$F$ & - & $0.40 \pm 0.08$ & $0.51 \pm 0.11$ \\
\hline$\chi_{\text {d.o.f. }}^{2}$ & 0.65 & 0.73 & $1.14 *$ \\
\hline
\end{tabular}

\section{Results and discussion}

Contrary to what was assumed in [22], it was shown in [31] that the Born terms play a comparable role to that played by the NLO contribution in the $K^{-} p \rightarrow K \Xi$ reactions. In spite of this result, similar goodness in reproducing the experimental data was reached by two models whose interaction kernels take into account the WT and the NLO terms, but they differ in the inclusion or not of the Born terms (see the corresponding values of $\chi_{\text {d.o.f. }}^{2}$ in table 3 ). Both models were fitted to the same experimental data [17, 24-27]. From the first two columns of table 3, one can appreciate the difference between the fitting parameters of the two models. As it was seen in [15, 18, 21], when the Born terms are included in the interaction kernel, one can obtain more 'natural sized' subtraction constants, despite the large associated errors. Actually, we are not able to gain anything in terms of accuracy and stability of the parameters of the model nor any improvement in the $\chi_{\text {d.o.f. }}^{2}$ can be seen, even after including the Born terms for which we have the certainty of their need to reproduce the $K \Xi$ channels properly.

A better understandig of the physics embeded in each of these two parametrizations emerges when splitting the $K^{-} p \rightarrow K \Xi$ cross-section into the isospin basis ( $I=0$ and $I=1$ components). This fact is ilustrated in figure 5 of [31] where it is shown that the $I=0$ components of these two models are in opposition with each other while the distribution of the $I=1$ components is almost coincident, although the strenght corresponding to WT+NLO+Born [31] is a little bit enhanced in the whole range. Such differences in the isospin components point towards the need of identifying 

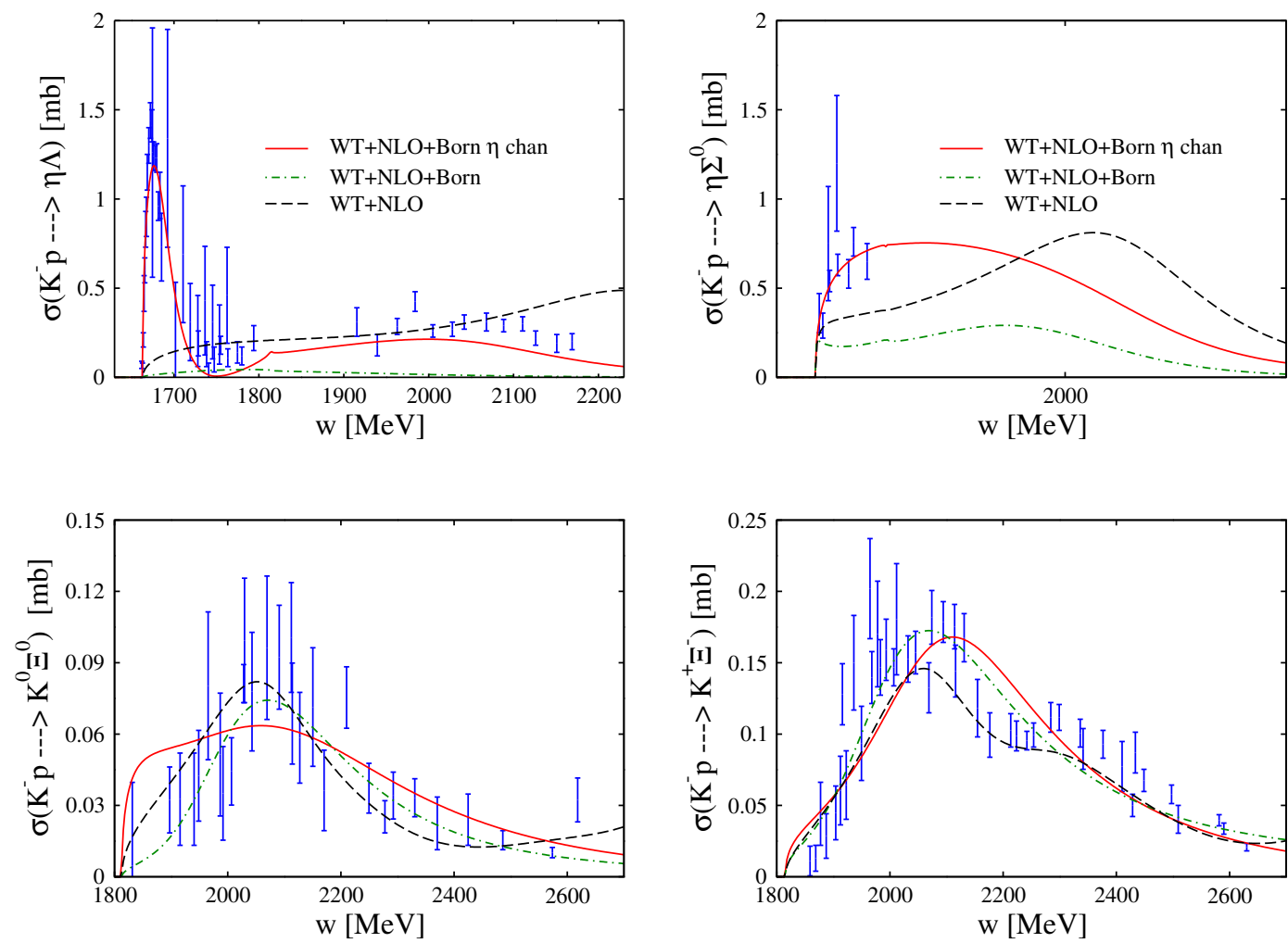

Figure 2. Total cross sections of the $K^{-} p \rightarrow \eta \Lambda, \eta \Sigma^{0}, K^{0} \Xi^{0}, K^{+} \Xi^{-}$reactions obtained from the NLO fit (dashed line), the WT+NLO+Born fit (dot-dashed line) and the WT+NLO+Born $\eta$ chan fit (solid line), see the text for more details. Experimental data are from $[27,36]$.

reactions that proceed through either $I=0$ or $I=1$, thereby acting as isospin selectors from which one can extract valuable information to better constrain the parameters of the meson-baryon lagrangian. Actually, since we study the meson-baryon interaction in the $S=-1$ sector, the most natural filtering processes are the $K^{-} p \rightarrow \eta \Lambda, \eta \Sigma^{0}$ reactions, which are of pure isospin 0 and 1 respectively. We therefore include the corresponding data [36] in a new fitting procedure in order to find more reliable values of the NLO coefficients and the rest of the parameters such as subtraction constants. The new fit consists of an interaction kernel which takes into account the WT, the Born and NLO terms and considers a larger amount of scattering data, which covers all the elastic and inelastic processes of the sector $[26,27,36]$ and the threshold observables cited previously $[17,24,25]$. The parameters of this fit, which is called WT+NLO+Born $\eta$ chan, are displayed in the third column of table 3 . From them, one can appreciate the homogeneity and accuracy achieved by the NLO coefficients. Another remarkable result is the obtention of natural sized values for all the subtraction constants. This leads us to believe that the inclusion of more experimental data from isospin filtering processes could favour the obtention of more reliable values for the low energy constants. Even though the models of table 3 can not be compared directly in terms of $\chi_{\text {d.o.f. }}^{2}$, the improvement of the agreement between experimental scattering data and the theoretical results is reflected in figure 2. In this figure, we only represent those cross sections for which appreciable changes from this new fit are seen. 
Non significant differences between the models are observed for the $K^{-} p \rightarrow \pi \Sigma, K N, \pi \Lambda$ reactions. From figure 2, we see that the cross sections of the $\eta$ channels can only be properly reproduced with the new fit performed (WT+NLO+Born $\eta$ chan), especially the $\Lambda(1670)$ resonant structure seen in $K^{-} p \rightarrow \eta \Lambda$. Concerning the $K \Xi$ channels, the three models give a similar good reproduction for the $K^{-} p \rightarrow K^{+} \Xi^{-}$cross section, but the old models (WT+NLO and WT+NLO+Born) clearly offer better agreement with the experiment than the new one for the $K^{-} p \rightarrow K^{0} \Xi^{0}$ cross section. In this last case, the inclusion of resonant terms by the same mechanism as [22] could be very helpful to accommodate the theoretical cross section to the experimental data. The $\Lambda(1890)$ might be a good candidate since is located at this energy region and is an isospin 0 resonance.
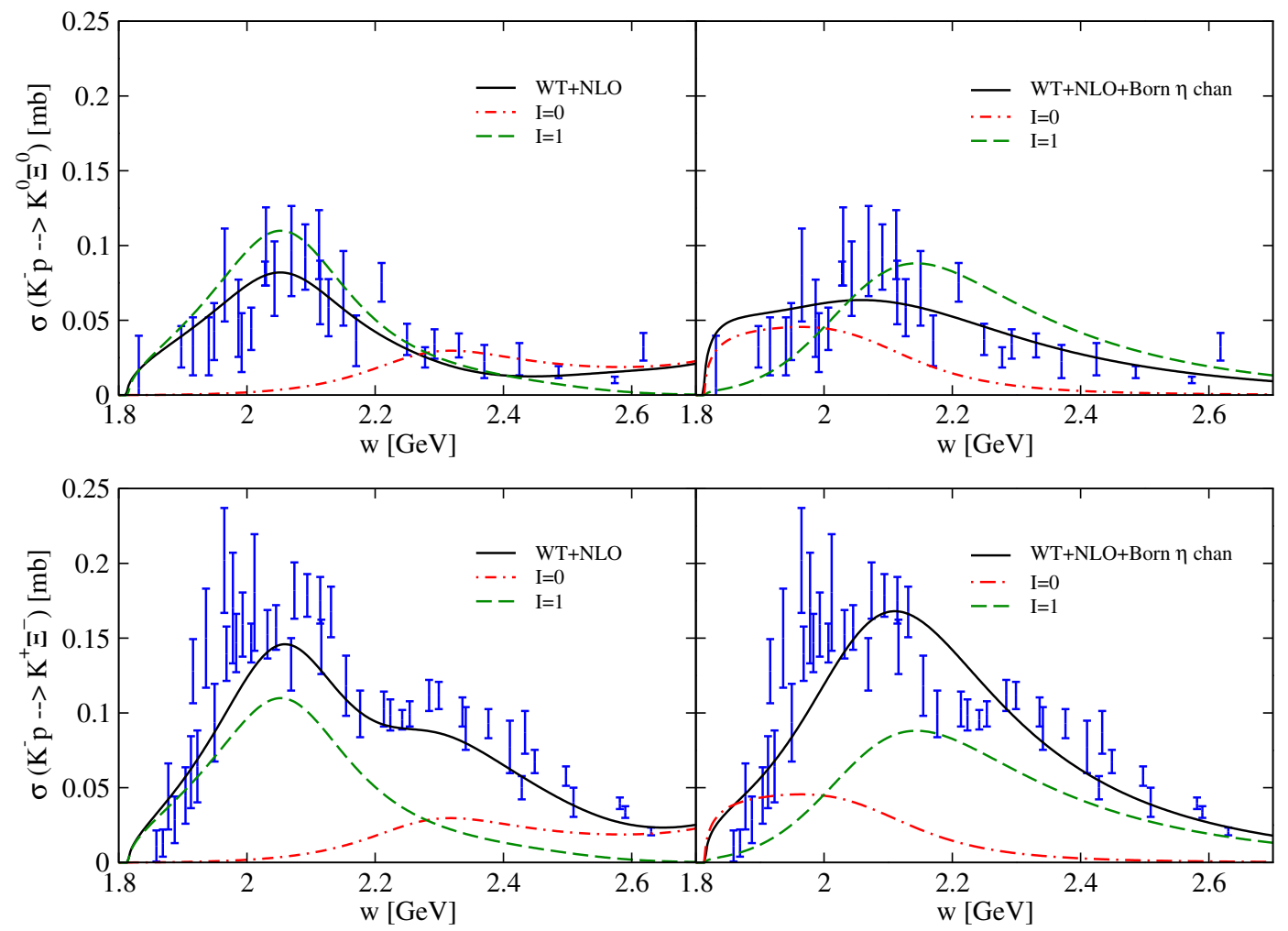

Figure 3. The total cross section data of the $K^{-} p \rightarrow K^{0} \Xi^{0}$ reaction is represented in the top pannels, where the left figure corresponds to the NLO model and the right one corresponds to the WT+NLO+Born $\eta$ chan model. The same distribution for the bottom pannels where the $K^{-} p \rightarrow K^{+} \Xi^{-}$cross section data are represented. The figure shows the complete results by means of solid lines, the results where only isospin $I=1$ component (dashed lines) or $I=0$ one (dot-dashed line) have been retained. Experimental data are taken from [27].

As a sample of the predictive power of the new model (WT+NLO+Born $\eta$ chan), we show in figure 4 the prediction for the $K^{-} n \rightarrow K^{0} \Xi^{-}$reaction, which is a pure $I=1$ process and which is scheduled to be mesured at Jlab [34]. To compare different models, we also include in the figure what is obtained by employing the WT+NLO+Born and NLO models, together with the experimental points, which have been divided by 2 to properly account for the size of the strangeness $S=-1$ component of the $K_{L}^{0}$. None of these two data points have been used in any fitting procedure. From 


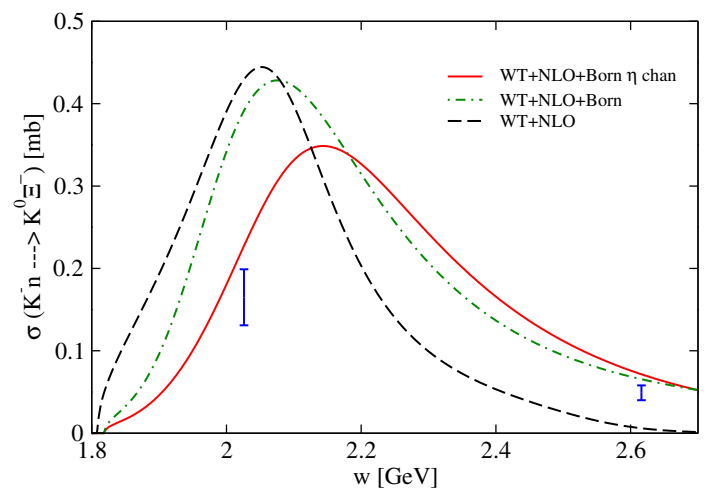

Figure 4. Total cross sections of the $K_{L}^{0} p \rightarrow K^{+} \Xi^{0}$ reactions for the for the WT+NLO+Born model (dot-dashed line) from [31], for the NLO model (dashed line) from [22] and for the the WT+NLO+Born $\eta$ chan (solid line). The experimental points of the $I=1 K^{-} n \rightarrow K^{0} \Xi^{-}$reaction, taken from [37] and divided by two, see [31] for more details.

figure 4, we can see that as the model accounts for more contributions in its interaction kernel and more data is included in the fit, especially data from isospin filtering processes, the predicted results get closer to the two available experimental points. Given these results, having more data from the proposed secondary $K_{L}^{0}$ beam at Jlab would be very helpful for constraining the theoretical models.

\section{Acknowledgements}

This work is partly supported by the Spanish Ministerio de Economia y Competitividad (MINECO) under the project MDM-2014-0369 of ICCUB (Unidad de Excelencia 'María de Maeztu'), and, with additional European FEDER funds, under the contract FIS2014-54762-P, by the Generalitat de Catalunya contract 2014SGR-401, and by the Spanish Excellence Network on Hadronic Physics FIS2014-57026-REDT.

\section{References}

[1] S. Scherer, Adv. Nucl. Phys. 27, 277 (2003)

[2] V. Bernard, N. Kaiser and U. G. Meissner, Int. J. Mod. Phys. E 4, 193 (1995)

[3] A. Pich, Rept. Prog. Phys. 58, 563 (1995)

[4] J. A. Oller, E. Oset and A. Ramos, Prog. Part. Nucl. Phys. 45, 157 (2000)

[5] N. Kaiser, P. B. Siegel, W. Weise, Nucl. Phys. A 594, 325 (1995)

[6] R. H. Dalitz and S. F. Tuan, Annals Phys. 8, 100 (1959); R. H. Dalitz and S. F. Tuan, Phys. Rev.

Lett. 2, 425 (1959); M. Jones, R. H. Dalitz and R. R. Horgan, Nucl. Phys. B 129, 45 (1977)

[7] N. Kaiser, T. Waas, W. Weise, Nucl. Phys. A 612, 297 (1997)

[8] E. Oset, A. Ramos, Nucl. Phys. A 636, 99 (1998)

[9] J. A. Oller, U. -G. Meissner, Phys. Lett. B 500, 263 (2001)

[10] M. F. M. Lutz, E. Kolomeitsev, Nucl. Phys. A 700, 193 (2002)

[11] B. Borasoy, E. Marco, S. Wetzel, Phys. Rev. C 66, 055208 (2002) 
[12] C. Garcia-Recio, J. Nieves, E. Ruiz Arriola and M. J. Vicente Vacas, Phys. Rev. D 67, 076009 (2003)

[13] D. Jido, J. A. Oller, E. Oset, A. Ramos and U. G. Meissner, Nucl. Phys. A 725, 181 (2003)

[14] A. Bahaoui, C. Fayard, T. Mizutani, B. Saghai, Phys. Rev. C 68, 064001 (2003)

[15] B. Borasoy, R. Nissler, W. Wiese, Eur. Phys. J. A 25, 79 (2005)

[16] B. Borasoy, U. -G. Meissner and R. Nissler, Phys. Rev. C 74, 055201 (2006)

[17] M. Bazzi, G. Beer, L. Bombelli, A. M. Bragadireanu, M. Cargnelli, G. Corradi, C. Curceanu (Petrascu) and A. d'Uffizi et al., Phys. Lett. B 704, 113 (2011)

[18] Y. Ikeda, T. Hyodo, W. Wiese, Nucl. Phys. A 881, 98 (2012)

[19] Zhi-Hui Guo, J. A. Oller, Phys. Rev. C 87, 035202 (2013)

[20] M. Mai and U. G. Meissner, Nucl. Phys. A 900, 51 (2013)

[21] T. Mizutani, C. Fayard, B. Saghai and K. Tsushima, Phys. Rev. C 87, 035201 (2013)

[22] A. Feijoo, V. K. Magas and A. Ramos, Phys. Rev. C 92, 015206 (2015)

[23] A. Ramos, A. Feijoo and V. K. Magas, Nucl. Phys. A 954, 58 (2016)

[24] R. J. Nowak et al., Nucl. Phys. B 139, 61 (1978)

[25] D. N. Tovee et al., Nucl. Phys. B 33, 493 (1971)

[26] J. K. Kim, Phys. Rev. Lett. 14, 89 (1965); T. S. Mast, et al., Phys. Rev. D 14, 13 (1976); R. O.

Bangerter, et al., Phys. Rev. D 23, 1484 (1981); J. Ciborowski, et al., J. Phys. G 8, 13 (1982)

[27] G. Burgun et al., Nucl. Phys. B 8, 447 (1968); J. R. Carlson, et al., Phys. Rev. D 7, 2533 (1973);

P. M. Dauber, et al., Phys. Rev. 179, 1262 (1969); M. Haque et al., Phys. Rev. 152, 1148 (1966); G.

W. London, et al., Phys. Rev. 143, 1034 (1966); T. G. Trippe, P. E. Schlein, Phys. Rev. 158, 1334 (1967); W. P. Trower, et al., Phys. Rev. 170, 1207 (1968)

[28] K. Nakayama, Y. Oh and H. Haberzettl, Phys. Rev. C 74, 035205 (2006)

[29] J. K. S. Man, Y. Oh and K. Nakayama, Phys. Rev. C 83, 055201 (2011)

[30] D. A. Sharov, V. L. Korotkikh and D. E. Lanskoy, Eur. Phys. J. A 47, 109 (2011)

[31] A. Ramos, A. Feijoo and V. K. Magas, Nucl. Phys. A 954, 58 (2016)

[32] A. Feijoo, V. K. Magas, A. Ramos and E. Oset, Phys. Rev. D 92, no. 7, 076015 (2015)

[33] V. K. Magas, A. Feijoo, A. Ramos and E. Oset These proceedings

[34] Letter of intent, Physics Opportunities with Secondary KL beam at JLAB, LOI-12-15-001

[35] T. Hyodo, D. Jido, Progress in Particle and Nuclear Physics 67, 55 (2012)

[36] A. Starostin et al. (Crystal Ball Collaboration), Phys. Rev. C64, 055205 (2001); D. F. Baxter et al., Nucl. Phys. B 67, 125 (1973); M. Jones et al., Nucl. Phys. B 90, 349 (1975); A. Berthon et al., Nuovo Cim. A 21, 146 (1974)

[37] J.P. Berge et al., Phys. Rev. 147, 945 (1966); S.A.B.R.E. Collaboration, J.C. Scheuer et al., Nucl. Phys. B 3361 (1971) 